\title{
The development of a mini-array for estimating the disease state of gastric adenocarcinoma by array CGH
} Tomoko Furuya ${ }^{1}$, Tetsuji Uchiyama², Atsushi Adachi ${ }^{2}$, Takae Okada ${ }^{1}$, Motonao Nakao ${ }^{1}$, Atsunori Oga ${ }^{1}$, Song-Ju Yang ${ }^{3}$, Shigeto Kawauchi ${ }^{1}$ and Kohsuke Sasaki*1

Address: ${ }^{1}$ Department of Pathology, Yamaguchi University School of Medicine, Ube 755-8505, Japan, ${ }^{2}$ Department of Surgery, Iwakuni Medical Center, Iwakuni 740-0021, Japan and ${ }^{3}$ Macrogen Inc, Seoul 153-081, Republic of Korea

Email: Tomoko Furuya - tfuruya@yamaguchi-u.ac.jp; Tetsuji Uchiyama - tetuuchi@silver.ocn.ne.jp; Atsushi Adachi - tetuuchi@silver.ocn.ne.jp; Takae Okada - takae@yamaguchi-u.ac.jp; Motonao Nakao - motonao@remus.dti.ne.jp; Atsunori Oga - oga@yamaguchi-u.ac.jp; SongJu Yang - sjyang@yahoo.co.jp; Shigeto Kawauchi - shig@yamaguchi-u.ac.jp; Kohsuke Sasaki* - kohsuke@yamaguchi-u.ac.jp

* Corresponding author

Published: 30 December 2008

BMC Cancer 2008, 8:393 doi:10.1 I86/147/-2407-8-393
Received: 6 December 2007

Accepted: 30 December 2008

This article is available from: http://www.biomedcentral.com/I47I-2407/8/393

(c) 2008 Furuya et al; licensee BioMed Central Ltd.

This is an Open Access article distributed under the terms of the Creative Commons Attribution License (http://creativecommons.org/licenses/by/2.0), which permits unrestricted use, distribution, and reproduction in any medium, provided the original work is properly cited.

\begin{abstract}
Background: The treatment strategy usually depends on the disease state in the individual patient. However, it is difficult to estimate the disease state before treatment in many patients. The purpose of this study was to develop a BAC (bacterial artificial chromosome) mini-array allowing for the estimation of node metastasis, liver metastasis, peritoneal dissemination and the depth of tumor invasion in gastric cancers.
\end{abstract}

Methods: Initially, the DNA copy number aberrations (DCNAs) were analyzed by array-based comparative genomic hybridization $(\mathrm{aCGH})$ in 83 gastric adenocarcinomas as a training-sample set. Next, two independent analytical methods were applied to the aCGH data to identify the BAC clones with DNA copy number aberrations that were linked with the disease states. One of the methods, a decision-tree model classifier, identified 6, 4, 4, 4, and 7 clones for estimating lymph node metastasis, liver metastasis, peritoneal dissemination, depth of tumor invasion, and histological type, respectively. In the other method, a clone-by-clone comparison of the frequency of the DNA copy number aberrations selected 26 clones to estimate the disease states.

Results: By spotting these 50 clones together with 26 frequently or rarely involved clones and 62 reference clones, a mini-array was made to estimate the above parameters, and the diagnostic performance of the mini-array was evaluated for an independent set of 30 gastric cancers (blinded - sample set). In comparison to the clinicopathological features, the overall accuracy was $66.7 \%$ for node metastasis, $86.7 \%$ for liver metastasis, $86.7 \%$ for peritoneal dissemination, and $96.7 \%$ for depth of tumor invasion. The intratumoral heterogeneity barely affected the diagnostic performance of the mini-array.

Conclusion: These results suggest that the mini-array makes it possible to determine an optimal treatment for each of the patients with gastric adenocarcinoma. 


\section{Background}

Gastric adenocarcinoma is one of the most frequent cancers worldwide, and it is the second leading cause of cancer death [1]. Although new diagnostic and therapeutic procedures are being developed, many patients still nevertheless die of the disease. The improvement of prognosis by the most optimal treatment is the first priority for all cancer patients. It is true that detection is critical to a rapid cure, though under the present circumstances gastric cancers are detected at various stages. The treatment strategy usually depends on the disease state in each individual patient. Surgery with a curative intent is applied to advanced cancers, while an endoscopic mucosal resection (EMR) or endoscopic submucosal dissection (ESD) is applied to early cancer without lymph node metastasis [2]. Accordingly, it is necessary to elucidate the disease state of an individual patient before any treatment or therapeutic procedure is started. In particular, an accurate evaluation of the nodal status and disease stage is critical in order to determine the appropriate treatment. However, the assessment of the disease state before treatment is not easy by conventional tests such as endoscopic inspection and microscopic examination of biopsy specimens. It is generally accepted that the biological characteristics of cancer are primarily dependent on the underlying genetic alterations of cancer cells. Therefore, a comprehensive analysis of the genomic changes in an individual type of cancer is necessary for identifying the genomic changes linked with the clinicopathological features. Microarray technology accomplishes this purpose. Arraybased comparative genomic hybridization (aCGH), a specific microarray method, allows the locus-by-locus measurement of DNA copy number aberrations (DCNAs) in cancer cells with a high resolution [3]. The aCGH has been applied to surgically removed gastric cancers to identify the chromosomal regions associated with carcinogenesis [4-9]. However, information concerning the relationship between DCNAs and the disease state is currently very limited. The identification of BAC clones with DCNAs that are linked with disease states may allow the exact estimation of disease states even in biopsy specimens at the time of the histological diagnosis, or before treatment. When a mini-array as a gastric cancer classifier that makes possible the quantitative measurement of genomic alterations is developed, it will be possible to estimate the disease state of each tumor for optimal treatment. The miniarray CGH may provide information concerning the disease states in order to determine the optimal treatment in individual cancer patients, thus contributing to personalized gastric cancer care.

In this study, the statistical analyses of aCGH data obtained from 83 sporadic gastric cancers identified 50 BAC clones linked with disease states including node metastasis, liver metastasis, peritoneal dissemination, the depth of tumor invasion and the histological type in gastric cancers. Next, using these BAC clones, a mini-array was constructed to evaluate the disease states of gastric cancer. In addition, the diagnostic performance of the mini-array was evaluated for an independent set of 30 gastric cancers. This is the first report of the development of a BAC mini-array which thus makes it possible to estimate the disease state in gastric cancer.

\section{Methods \\ Tissue specimens and DNA samples}

Eighty-three consecutive surgically removed gastric adenocarcinomas were evaluated for the selection of BAC clones linked with the clinicopathological features. They included 10 early cancers and 73 advanced cancers. The patients consisted of 62 males and 21 females with an average age of 70 years ranging from 44 to 89 years old. The family histories were noncontributory for all patients and all tumors were considered to be sporadic. The clinicopathological features are summarized in Table 1. In brief, according to Lauren's histological classification, 41 tumors were classified as intestinal-type gastric cancer, while the others were diffuse-type cancers. Node and liver metastases were detected in 60 and 6 cancers, respectively. In this series, 19 tumors showed a peritoneal dissemination of the cancer cells. The tissue specimens were stored at $-80^{\circ} \mathrm{C}$ until use. A tissue microdissection technique was used to reduce the contamination of the normal tissue components for the array CGH analyses, as previously described [10]. High molecular weight genomic DNA was extracted from the microdissected tumor tissue specimens with a DNA extraction kit (SepaGene, Sankojunyaku Co., Ltd, Tokyo, Japan) according to the manufacturer's instructions [11-13]. Control DNA (Promega, Madison, WI) was used as a reference. The study protocol was conducted under the approval of the Institutional Review Board for Human Use at the Yamaguchi University School of Medicine in 2004, and informed consent for this study was obtained from every patient.

\section{Array CGH for screening}

The array CGH experiments were performed with a MacArray $^{\mathrm{TM}}$ Karyo 1400 (Macrogen Inc., Seoul, Korea) according to the manufacturer's protocol http:// www.macrogen.co.kr/eng/biochip/karyo summary.jsp, which provided the BAC chip information together with information of the end-sequenced BAC clones and data processing methods, as previously described [14-16]. The arrays consisted of triplicate spotted 1,440 human bacterial artificial chromosome (BAC) clones, including 356 cancer-related genes, which covered the whole human genome at an average interval of $2.3 \mathrm{Mb}$. Sample and gender matched reference genomic DNAs (500 ng each) were labeled by the random priming method with fluorescence dyes, Cy 3 and Cy 5, respectively. The labeled DNAs were 
Table I: Clinicopathological summary of 83 gastric adenocarcinomas

\begin{tabular}{|c|c|c|}
\hline \multicolumn{3}{|l|}{ For Screening } \\
\hline The number of gastric cancers examined: & 83 & \\
\hline Average age of patients (range): & 70 years old ( $44-89$ years) & \\
\hline \multicolumn{3}{|l|}{ Histological type of gastric cancers } \\
\hline Intestinal-type: & & \\
\hline \multirow[t]{2}{*}{ Diffuse-type: } & & \\
\hline & Intestinal-type & Diffuse-type \\
\hline Average age (years) & 72 & 68 \\
\hline $\operatorname{Sex}(F / M)$ & $9 / 32$ & $12 / 30$ \\
\hline Node metastasis & 26 & 34 \\
\hline Liver metastasis & 3 & 3 \\
\hline Peritoneal dissemination & 7 & 12 \\
\hline Early/advanced cancers* & $6 / 35$ & $4 / 38$ \\
\hline \multicolumn{3}{|l|}{ For validation of the mini-array } \\
\hline The number of gastric cancers examined: & 30 & \\
\hline Average age of patients (range): & 69.3 years old ( $44-88$ years) & \\
\hline $\operatorname{Sex}(F / M)$ & $11 / 19$ & \\
\hline \multicolumn{3}{|l|}{ Histological type of gastric cancers } \\
\hline Intestinal/diffuse & & \\
\hline Node metastasis & & \\
\hline Liver metastasis & & \\
\hline Peritoneal dissemination & & \\
\hline Early/advanced cancers* & & \\
\hline
\end{tabular}

*Early gastric cancer is defined as a tumor with invasion limited to mucosa or submucosa according to the Japanese classification of gastric carcinoma (9).

mixed with Cot-1 DNA (50 $\mu$ g, Gibco BRL, Gaithersburg, $\mathrm{MD})$ and then were hybridized to the array slides for 2 days at $37^{\circ} \mathrm{C}$ in a moist chamber. The array slides were rinsed in a washing buffer and dried well. The array slides were scanned with a Gene Pix 4000A scanner (Axon Instruments, Union City, CA). The fluorescence images were analyzed using the MAC Viewer ${ }^{\mathrm{TM}}$ software program (Macrogen Inc.) optimized for the analysis of the array as previously reported [14-16]. The fluorescence spots were defined with the automatic grid feature and adjusted manually. All CGH ratios were automatically converted to log base 2 . The ratios of the fluorescence intensities of all spots were plotted against the distance of the clones along the chromosomes. For each BAC clone, the average ratios that deviated significantly from zero were considered to be abnormal $\left( \pm \log _{2} 0.25\right)$, and almost all spots were within the area between the cutoff lines $\left( \pm \log _{2} 0.25\right)$ for male/female DNA samples. In addition, we defined $\log _{2}$ ratio $>1.0$ as amplifications.

\section{Array CGH data analysis}

The aCGH data of the 83 gastric cancers were analyzed by two independent methods referred to as method 1 and method 2 for convenience. In method 1, a WEKA decision-tree model classifier, J48 http:// www.cs.waikato.ac.nz/ml/weka/[17] was applied to the aCGH data to identify the BAC clones and their copy numbers for differentiating between gastric cancers with and without node metastasis, with and without liver metastasis, with and without peritoneal dissemination, between early and advanced cancers, and between intestinal type and diffuse type cancers. In method 2, the cloneby-clone frequency of the DCNAs between the two groups, e.g., between cancer with and without node metastasis, was compared using the $\chi^{2}$-test to identify the BAC clones that were used for the distinction between two groups. The difference was considered significant when its P-value was less than 0.05 , and the BAC clones with a Pvalue of less than 0.01 were used for the fabrication of a mini-array specific for gastric cancer.

\section{Customization of BAC mini-array specific for gastric cancer}

The mini-array was made using 50 BAC clones chosen by the two analytical methods of the aCGH data from 83 gastric cancers to estimate node and liver metastases, peritoneal dissemination, depth of tumor invasion, and histological type; the method 1 identified 24 BAC clones of which one was shared between node metastasis and liver metastasis, and the method 2 identified 26 clones. In addition to these BAC clones, the mini-array also contained 26 clones with frequent or infrequent DCNAs and 62 reference clones. A total of 138 BAC clones were spotted in triplicate on two discrete parts of a glass slide, and therefore, each slide was used for two specimens (Fig 1b). 


\section{Validation of performance for mini-array}

The performance of the mini-array was evaluated for node metastasis, liver metastasis, peritoneal dissemination, depth of invasion, and histological type of gastric cancers for an independent set of 30 gastric cancers (Table 1 ). The cutoff value was also applied to data analysis of the miniarray. The copy numbers for 138 BAC clones were compared between the screening arrays and the mini-arrays. When the diagnosis was different between the two methods, priority was given to the diagnosis that indicated a more advanced stage in the evaluation of the mini-array.

\section{Effects of intratumoral heterogeneity on the performance of mini-array}

The effects of the intratumoral genomic heterogeneity on the performance of the mini-array in the randomly selected five gastric tumors were examined. Four tissue specimens were taken from different parts of a tumor. The procedures for tissue microdissection, DNA extraction, aCGH and the data analysis were as noted above.

\section{Results}

The DCNAs were detected by aCGH for the multiple BAC clones in all gastric adenocarcinomas. Although the number of BAC clones with DCNA varied from tumor to tumor, roughly $15 \%$ of clones showed DCNAs for each tumor. Overall, frequent DNA copy number gains were detected for chromosomal regions 20q12-q13 (at the frequency of $43 \%)$, and $8 \mathrm{q} 24(43 \%)$, and frequent DNA copy number losses were detected for chromosomal regions $4 \mathrm{q} 35.2(37 \%), 1 \mathrm{p} 36(36 \%), 4 \mathrm{q} 34$ (36\%), 4q12 (35\%), 14q32 (33\%), and 22q11 (34\%), in addition to the Y chromosome (Fig 1a and see Additional file 1).

The comparison of the aCGH data with the clinicopathological features of the tumors using the decision-tree classifier (method 1) allowed the identification of the BAC clones for the differentiation between gastric cancers with and without specific clinicopathological characters. The gastric cancers with node metastasis were distinguished from those without it with an accuracy of $100 \%$ by examining the extent of the DCNAs for the six BAC clones mapped to 5q13.2, 13q31.1, 1p22.3, 1p34.2, 14q32.2, and 3q13.12 (Fig 2a). The procedures of the analysis were as follows: The DNA copy number of a tumor at $5 \mathrm{q} 13.2$ was applied to the criterion of the first clone. If the DNA copy number of the clone was $\%-0.349$, the tumor was classified as a tumor without node metastasis. Six of 83 tumors were classified into this group. When the DNA copy number of the tumor was not the case at the first clone, the second criterion (>-0.37) was checked. Five tumors were separated from the remaining 77 tumors. When the DNA copy number of the clone at 13q31.1 was $\% 0.37$ in the tumor, then the third clone located on 1 p22.3 was examined. If the DNA copy number of the tumor met the criterion of the third clone, \% -0.042 , nodal metastasis was considered positive in this tumor. Thirty-six cancers were classified into this category. In this way, the DNA copy number of the tumor was in turn examined from the first to the sixth clone. Eventually, all gastric cancers were classified into either of the two groups, cancers with and without node metastasis.

Gastric adenocarcinomas with liver metastasis were separated from those without it at a $100 \%$ accuracy rate by checking the DNA copy number for the four BAC clones mapped to 1q44-qter, 5q13.2, 6q23.2, and 7q31.1, as shown in Fig 2b. The high level loss of 5q13.2 was detected in tumors with neither node nor liver metastasis. The decision-tree classifier also permitted the classification of gastric cancer into either a tumor with or without peritoneal dissemination at the correct classification rate of $98.8 \%$ by the copy number alterations of the four BAC clones mapped to 4q13.3, 4q32.2, 13q12.13, and $12 q 24.23$ (Fig 2c). The decision-tree classifier made it possible to divide the gastric cancers into two groups, early and advanced cancers, on the basis of the DNA copy number of the four BAC clones mapped to 1q43, 21q22.2, 16q22.1, and 6p21.1 (Fig 2d). In addition, the examination of the seven BAC clones made it possible to accurately differentiate between these two histologic types, the intestinal-type and the diffuse-type (Fig 2e). A DNA copy number change in a clone located in 5q13.2 was linked with both node and liver metastasis, and in total 24 clones were identified by method 1 .

A statistical analysis of the aCGH data was also performed using the protocol referred to as method 2 to identify the clones for classifying the gastric cancers into two groups with opposite characteristics. The comparison of the frequency of the DCNAs clone-by-clone revealed many BAC clones with statistical differences in their frequency between the tumors with and without node metastasis, between the tumors with and without liver metastasis, between the tumors with and without peritoneal dissemination, and between the intestinal- and diffuse-type cancers. Seven, six, five, and eight BAC clones, for a total of 26 clones, were selected to evaluate for node metastasis, liver metastasis, peritoneal dissemination, and histological type, respectively (Table 2 ).

DNA amplification was detected at chromosomal regions 17q21.1(harboring HER-2), 11q13.3 (CCND1), and $11 \mathrm{q} 13$ (FGF4) in four, three, and three tumors, respectively, though the amplification was not correlated with any clinicopathological feature of the gastric cancers.

\section{Validation of the mini-array}

The dye-swap hybridization experiments revealed that the switching of the dyes did not affect the aCGH profiles (Fig 


\section{a}
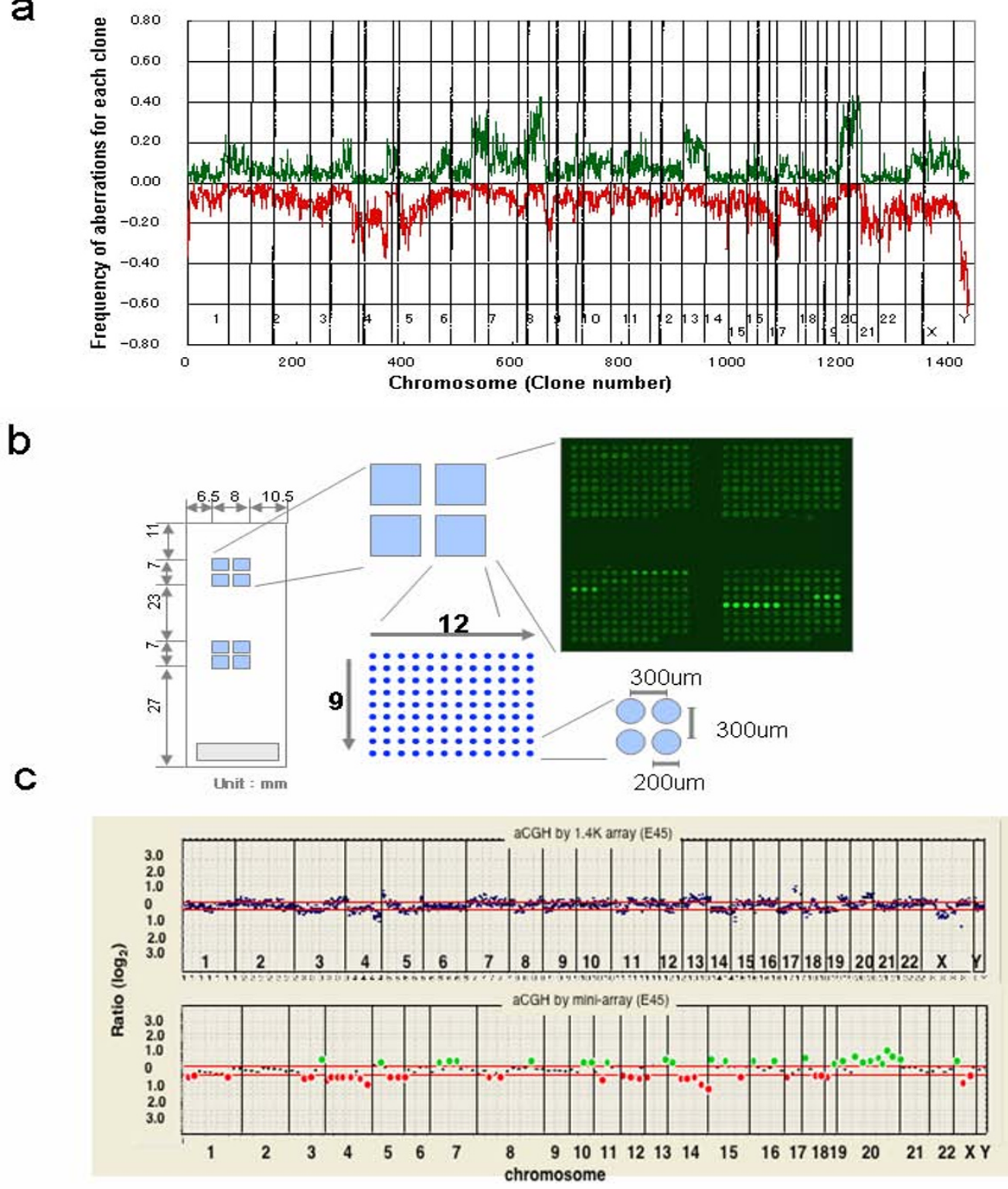

\section{Figure I}

(a) Frequency of gains and losses in $\mathbf{8 3}$ gastric cancers. A green line denotes a copy number gain and a red line denotes a copy number loss. Gains of $7 p, 8 q$, and $20 q$, and losses of $4 q, 17 p$, and 21 are frequent in gastric adenocarcinoma. (b-left) Design of a mini-array. The mini-array spotted with 50 BAC clones chosen by two analytical methods of the aCGH data from 83 gastric cancers to estimate the node and liver metastases, peritoneal dissemination, depth of tumor invasion, and histological type, 26 clones with frequent or infrequent DCNAs, and 62 reference clones. A total of I 38 BAC clones were spotted in triplicate on two discrete parts of a glass slide, and therefore, each slide was used for two specimens. The diameter of each spot is approximately $200 \mu \mathrm{m}$. (b-right) A hybridization image for the mini-array. Note the same color image for three spots each in the mini-array. (c) A comparison of aCGH profiles between the screening chip (upper) and the mini-array (lower) for a case of gastric adenocarcinoma. A profile of aCGH in the mini-array basically replicates that in the screening array. The red lines indicate $\log _{2} 0.25$ and $-\log _{2} 0.25$, respectively. The green and red spots indicate the BAC clones with a copy number gain and loss, respectively. 
$3)$. The diagnostic performance of the mini-array was evaluated for the independent set of 30 gastric cancers. Although the density of the dots was less in the mini-array than in the screening $1.4 \mathrm{~K}$ arrays, the data by the miniarray analysis were virtually equivalent to those by the screening array (Fig 1c). The copy number of each clone in the mini-array correlated well with that of the corresponding clone in the screening array (average correlation coefficient $r=0.747$, ranging from 0.664 to 0.920 ). First, the mini-array data analysis was made regardless of the clinicopathological features, and then, the aCGH data from the mini-array were checked out against the clinicopathological features of an individual tumor. The diagnostic accuracy was considerably high even in a single analysis method and was not significantly different between the two methods, as shown in Table 3. However, the combination of the two analytical methods slightly improved the accuracy for all parameters except for the histological typing (Table 3). The overall diagnostic accuracy was as follows: $66.7 \%$ (sensitivity; 0.95 , and specificity; 0.0 ) for node metastasis, $86.7 \%$ (sensitivity; 0.67 and specificity; 0.89 ) for liver metastasis, $86.7 \%$ (sensitivity; 0.20 , and specificity; 1.0) for peritoneal dissemination, and 96.7\% (sensitivity; 1.0, and specificity 0.67 ) for the depth of tumor invasion (Table 3). Nine of ten cancers misclassified by the mini-array were false positives, and one of them was a false negative with regard to the evaluation of node metastasis. As for the estimation of liver metastasis, four cancers were misclassified; three of them were false positives and one was a false negative. As for the estimation of peritoneal dissemination, all of the misclassified cancers were false negatives (Table 3 ). As for the estimation of the depth of tumor invasion, only a single case was misclassified by the mini-array and it was overdiagnosed (Table 3).

\section{Effects of intratumoral heterogeneity on the diagnostic performance of the mini-array}

A slight variation in the DNA copy number of the BAC clone was observed between the samples taken from a single tumor. Regardless of whether the samples provided a correct diagnosis, however, the diagnosis hardly differed between the four samples from the same tumor (Table 4). In particular, there were no BAC clones with a copy number variation affecting the estimation of liver metastasis and peritoneal dissemination in all tumors. Occasionally there were tumors in which one or two clones affecting the diagnosis were present for one or two items (Table 4). In one early cancer (invasion into the submucosal layer), however, one of four tissue specimens correctly estimated the tumor as an early cancer, while the others estimated it as an advanced cancer. In another case, three of the four tissue specimens correctly estimated a tumor as an advanced cancer, while the other estimated it as an early cancer. However, the overall diagnostic accu- racy (in the combination of methods 1 and 2) was virtually unaffected by the intratumoral heterogeneity.

\section{Discussion}

The evaluation of the disease state before treatment is necessary to appropriately select the optimal treatments with a high therapeutic efficiency and high quality of life for each patient with gastric cancer. With this view, the genomic changes characterizing gastric cancers have been investigated [5-10,13]. The chromosomal regions with frequent copy number aberrations in gastric cancers were easily detected by aCGH $[5,7,18,19]$, and some of them were reported as a genomic marker associated with peritoneal dissemination [20] and node metastasis of gastric cancer $[6,18,19,21]$. However, the examination of only a few genomic markers is insufficient for the precise estimation of the disease state. Alternatively, the combination of the genomic markers with a strong diagnostic impact may allow for the precise estimation of the disease state in each case. The present study demonstrated that the comparison of the DNA copy number of each BAC clone between the two groups of tumors with opposite characteristics identified the BAC clones linked with the disease states including node metastasis, liver metastasis, peritoneal dissemination, depth of tumor invasion, and histological type of gastric cancer. Two independent data analysis methods selected the BAC clones allowing for the reliable classification of 83 gastric cancers (training samples) into two tumor groups with opposite characteristics. In total, 50 BAC clones were selected as genomic markers for estimating the disease states of the gastric cancers. With the exception of a single clone located in $5 \mathrm{q} 13.2$ these clones were associated with only a single characteristic of the disease states. The copy number loss of the clone in 5q13.2 was frequent in the gastric cancers with neither node nor liver metastasis, but the converse was not true. Therefore, although the genetic mechanisms are partly shared by the lymph node and liver metastases of gastric cancer, it is convenient to think that the genomic alterations associated with tumor metastasis are basically different between the target organs, as explained by the well-known 'seed and soil' theory. The BAC clones involved in tumor metastasis are obviously different from the clones involved in the peritoneal dissemination of tumors. These BAC clones identified can be biomarkers to estimate the disease state by definition [22]. At present, however, it is uncertain whether the genes located in these BAC clones are truly involved in the invasion, metastasis or dissemination of gastric cancer, and how the genes participate in the node metastasis, liver metastases or peritoneal dissemination of gastric cancer.

Microarray technologies are being applied to cancer diagnosis and the prognostic prediction in malignancies. A microarray-based gene expression analysis has been pro- 


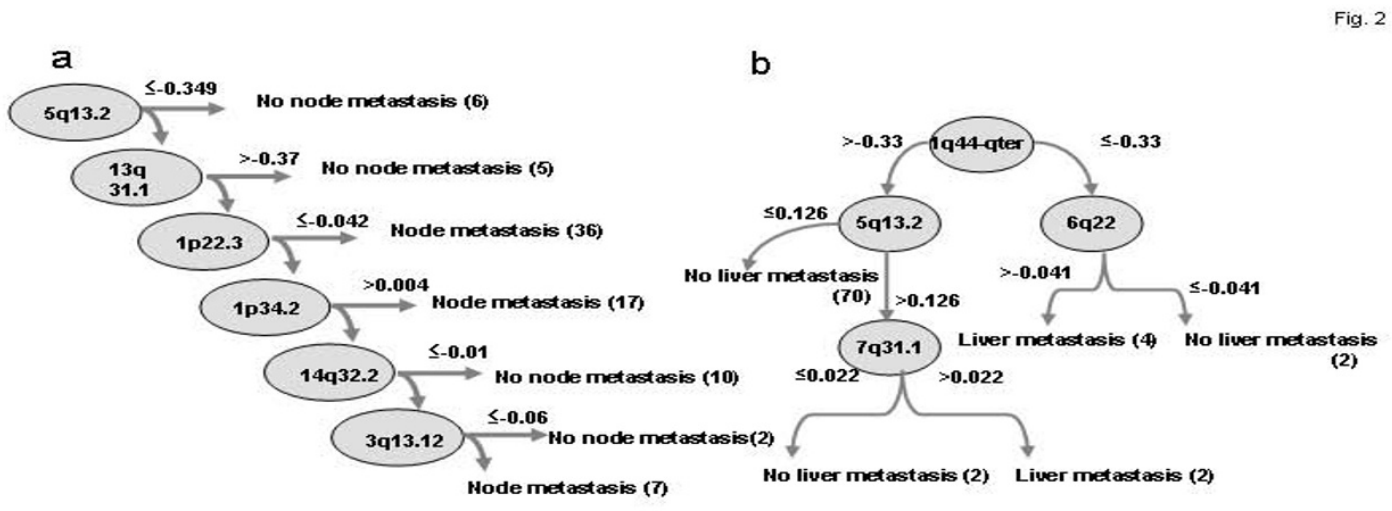

$\mathrm{c}$

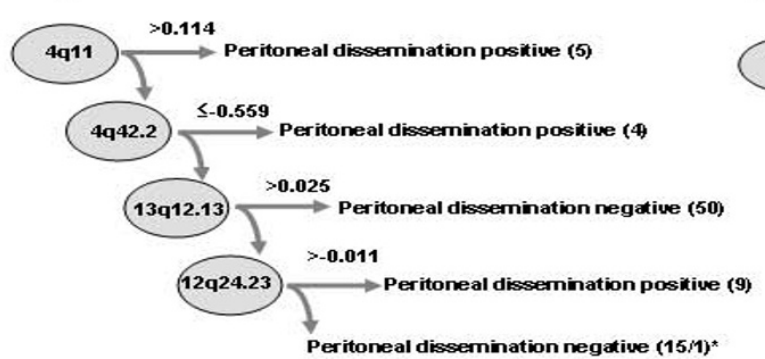

d

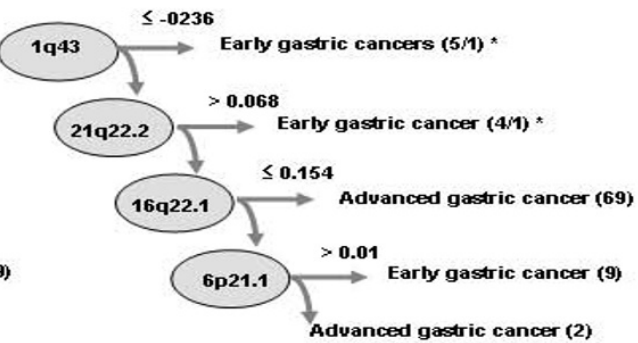

e *One tumor is incorrectly classified

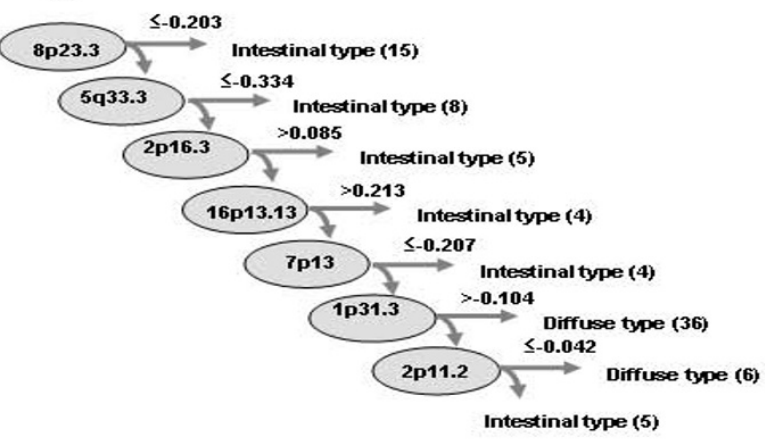

Figure 2

Identification of BAC clones by a decision-tree algorithm to classify the gastric cancers into two groups with different characters. (a) Differentiation between gastric cancers with and without node metastasis. These two groups can be clearly differentiated by examining the degree of the copy number changes of six BAC clones mapped to $5 \mathrm{q} I 3.2$, I3q3I.I, Ip22.3, I 34.2, I4q32.2, and 3qI3.12 in descending order. A tumor of which the copy number at $5 q \mid 3.2$ was $\leq-0.346\left(\log _{2}\right)$ shows no node metastasis. When the DNA copy number of the tumor was not the case at the first clone, the second criterion $(>-0.37$ at $13 q 3 \mathrm{I} . \mathrm{I})$ was checked. Five tumors were separated from the remaining 77 tumors at this point. When the DNA copy number of the clone at I $3 \mathrm{q} 3 \mathrm{I}$.I was $\% 0.37$ in the tumor, the third clone located on Ip 22.3 was examined. If the DNA copy number of the tumor meets the criterion of the third clone, $\%-0.042$, nodal metastasis was positive in this tumor. Thirtysix cancers were classified into this category at this point. In this way, the DNA copy number of the tumor was in turn examined from the first to the sixth clone. Each step successively sorts a cluster of either group. Eventually, all gastric cancers were classified into either of two groups, cancers with and without node metastasis. The figures in parentheses indicate the number of tumors fitting the requirements. The correctly classified instances were 83 (100\%), and the incorrectly classified instances were $0(0 \%)$. In this classifier, the number of leaves is seven, and the size of the tree was 13 . In the same way as the case of node metastasis, BAC clones and their copy numbers were determined for liver metastasis (b), peritoneal dissemination (c), depth of tumor invasion (early or advanced cancer)(d), and histologic type (e). 
Table 2:

\begin{tabular}{|c|c|c|c|c|c|}
\hline BAC ID & Chrom. region & Candidate Gene & Frequency of DCNAs(\%) & Frequency of DCNAs(\%) & P-value* \\
\hline \multicolumn{6}{|l|}{ Gain } \\
\hline Node metastasis & & & Positive $(n=60)$ & Negative $(n=23)$ & \\
\hline 225 & $2 q 24.32$ & TMEMI32D & 3 & 26 & 0.0005 \\
\hline 1308 & $5 q 13.2$ & $\mathrm{FCHO} 2, \mathrm{MGCl} 3034$ & 7 & 43 & 0.0005 \\
\hline 491 & $16 \mathrm{p} \mid 3.11$ & PKDIP3 & 5 & 35 & 0.0006 \\
\hline 884 & $19 q 13.32$ & N.i. & 5 & 30 & 0.0006 \\
\hline Liver metastasis & & & Positive $(n=6)$ & Negative $(n=77)$ & \\
\hline 1322 & $|p| 3.2$ & CHIA, Clorf88, OVGPI & 43 & 3 & $<0.0001$ \\
\hline $127 \mid$ & $8 p 22$ & N.i. & 67 & 13 & 0.0016 \\
\hline 92 & $11 p \mid 5$ & ST5 & 43 & 6 & 0.0006 \\
\hline Peritoneal dissemination & & & Positive $(n=19)$ & Negative $(n=64)$ & \\
\hline 948 & $15 q 22.1$ & MYOIE, LDHAL6B & 22 & 0 & 0.0002 \\
\hline 155 & Xp2I.2 & $\mathrm{XK}$ & 35 & 5 & 0.0009 \\
\hline Histologic type & & & Diffuse $(n=42)$ & Intestinal $(n=4 I)$ & \\
\hline 563 & $10 p \mid 5.3$ & DIP2C & 7 & 38 & 0.0011 \\
\hline 911 & $10 \mathrm{q} 24.2$ & SPFHI, CHUK, CWFI9LI & 0 & 23 & 0.0013 \\
\hline \multicolumn{6}{|l|}{ Loss } \\
\hline Node metastasis & & & Positive $(n=60)$ & Negative $(n=23)$ & \\
\hline 936 & $8 p 21.2$ & DOCK5, GNRHI, KCTD9, CDCA2 & 3 & 23 & 0.0023 \\
\hline 1158 & $8 p 21.1$ & EXTL3 & 3 & 21 & 0.0023 \\
\hline 878 & $13 q 31.2$ & SLITRK5 & 13 & 47 & 0.0007 \\
\hline Liver metastasis & & & Positive $(n=6)$ & Negative $(n=77)$ & \\
\hline 877 & Ip32.3 & LRP8, & 50 & 5 & 0.0006 \\
\hline 1386 & $7 q 35$ & CNTNAP2, & 43 & 4 & 0.0001 \\
\hline 309 & $21 \mathrm{q} 22.3$ & HSF2BP, KIAA0I79, & 29 & 1 & 0.0002 \\
\hline Peritoneal dissemination & & & Positive $(n=19)$ & Negative $(n=64)$ & \\
\hline 1407 & $3 p 26.3$ & CNTN6 & 33 & 6 & 0.0009 \\
\hline 503 & $3 p 24.3$ & UBE2EI & 32 & 6 & 0.0009 \\
\hline 229 & $4 q 31.1$ & MAML3 & 44 & 5 & $<0.0001$ \\
\hline Histologic type & & & Diffuse $(n=42)$ & Intestinal $(n=4 I)$ & \\
\hline 1292 & $4 q 34.1$ & N.i. & 17 & 53 & 0.0004 \\
\hline 1393 & $5 q 33.2$ & TIMD4 & 15 & 48 & 0.0007 \\
\hline 91 & $8 p 21.3$ & INTSIO & 0 & 24 & 0.0006 \\
\hline 227 & $14 q 32.33$ & IGHV3-22 & 16 & 54 & 0.0001 \\
\hline 1122 & $15 q 15.3$ & GANC, CAPN3 & 2 & 27 & 0.0003 \\
\hline 1210 & $|7 q| 3.2$ & ATP2A3, ZZEFI & 5 & 41 & 0.0001 \\
\hline
\end{tabular}

N.I.; not identified

$* \chi^{2}$ test between two groups

Ratios $\left(\log _{2}\right)$ that exceed $>0.25$ are considered DNA copy number gains, and ratios $\left(\log _{2}\right)$ that exceed $<-0.25$ are considered to be DNA copy number losses.

posed to estimate the prognosis of cancer patients $[23,24]$ and a small number of specific microarrays have been developed for this purpose [22,23].
In this study, a gastric cancer specific mini-array was spotted with 138 BAC clones including 50 clones chosen by the screening of 83 cancers. The number of spots in the

Table 3: Diagnostic accuracy of the mini-array for an independent series of $\mathbf{3 0}$ gastric cancers

\begin{tabular}{|c|c|c|c|c|}
\hline & Node Metastasis & Liver Metastasis & Peritoneal Dissemination & Advanced cancers \\
\hline \multicolumn{5}{|l|}{ Accuracy } \\
\hline Method I & $19 / 30(63.3 \%)$ & $27 / 30(90.0 \%)$ & $25 / 30(83.3 \%)$ & $29 / 30(96.6 \%)$ \\
\hline Method 2 & $20 / 30(66.7 \%)$ & $26 / 30(86.7 \%)$ & $26 / 30(86.3 \%)$ & - \\
\hline Overall Accuracy & $20 / 30(66.7 \%)$ & $26 / 30(86.7 \%)$ & $26 / 30(86.7 \%)$ & $29 / 30(96.7 \%)$ \\
\hline Sensitivity & $20 / 21(0.95)$ & $2 / 3(0.67)$ & $\mathrm{I} / 5(0.20)$ & $27 / 27(1.0)$ \\
\hline Specificity & $0 / 9(0.0)$ & $24 / 27(0.89)$ & $25 / 25(1.0)$ & $2 / 3(0.67)$ \\
\hline False positive & $9 / 9(1.0)$ & $3 / 27(0.11)$ & $0 / 25(0.0)$ & I (over-diagnosis) \\
\hline False negative & $\mathrm{I} / 2 \mathrm{I}(0.05)$ & $\mathrm{I} / 3(0.33)$ & $4 / 5(0.80)$ & 0 (under-diagnosis) \\
\hline
\end{tabular}




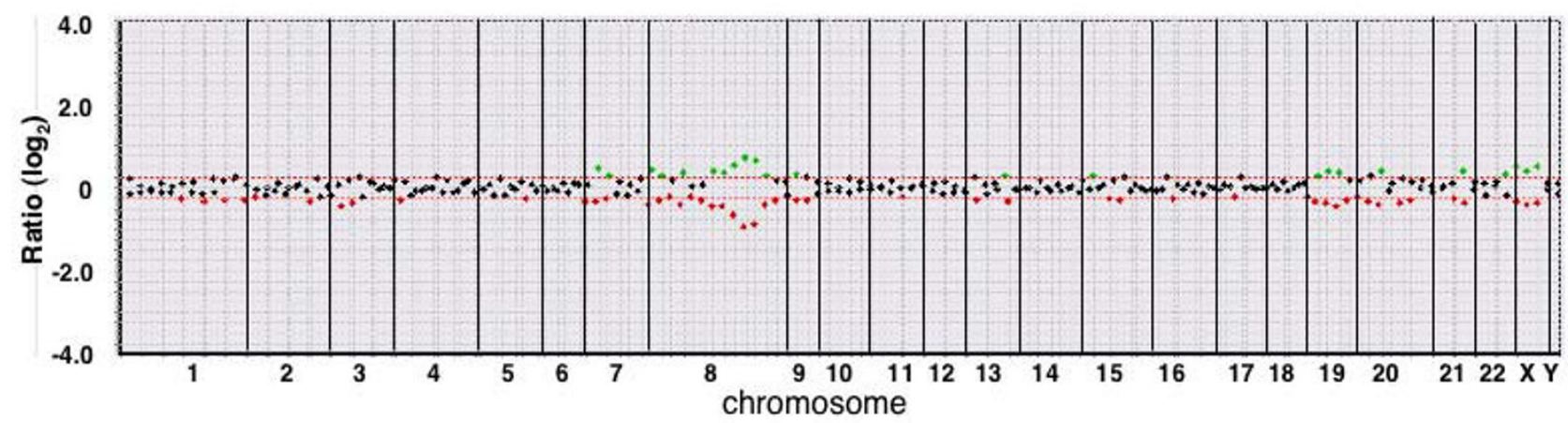

Figure 3

Array CGH profiles of a case (J9I) of gastric cancer in the dye-swap hybridization experiments for the miniarray. The tumor DNA is labeled with Cy3 or Cy5, and the reference DNA is labeled with Cy5 or Cy3, respectively. Each data point along the ratio plot represents the normalized ratio of the individual clones linearly ordered from chromosome $\mathrm{I}$ to the $\mathrm{Y}$ chromosome. The intensity ratios above or below the threshold are depicted as green or red dots, respectively. Two profiles show a mirror state, though a slight discordance may be present between some dots. The copy number gain is unambiguous for the BAC clones in chromosome 8 in this case.

mini-array was reduced to one-tenth in comparison to the original $1.4 \mathrm{~K}$ arrays that were used for the screening of the DNA copy number changes in this study. Furthermore, the number of spotted probes was much less in the present mini-array than in the gene expression type arrays such as the MammaPrint [25]. The mini-array developed in this study allowing the estimation of the disease states based on the DCNAs is unique. This is the first report of the development of a BAC mini-array in order to estimate the disease state of cancer. The diagnostic performance of

Table 4: Intratumoral genomic heterogeneity and diagnostic performance of the mini-array

\begin{tabular}{lllllll}
\hline \multicolumn{9}{c}{ Case No. } & Total \\
\cline { 2 - 5 } & S39 & B17 & C22 & 187 & S50 &
\end{tabular}

\begin{tabular}{lcccccc}
\hline Method I & & & & & & \\
Node metastasis & - & $+(I)$ & - & - & $+(2)$ & $3 / 20$ \\
Liver metastasis & - & - & - & - & - & $0 / 20$ \\
Peritoneal dissemination & - & - & - & - & - & $0 / 20$ \\
Early or advanced & $+(I)$ & - & - & $+(I)$ & - & $2 / 20$ \\
Histologic type* & $+(I)$ & - & - & - & $+(2)$ & $3 / 20$ \\
Method 2 & & & & & & \\
Node metastasis & - & - & - & $+(I)$ & - & $1 / 20$ \\
Liver metastasis & - & - & - & - & - & $0 / 20$ \\
Peritoneal dissemination & - & - & - & - & - & $0 / 20$ \\
Histologic type* & - & - & - & $+(I)$ & - & $1 / 20$ \\
& & & & & &
\end{tabular}

The difference in diagnosis was rare between four samples from the same tumor. In particular, there were no BAC clones affecting the estimation of liver metastasis and peritoneal dissemination in all of the samples.

-; Absence of intratumoral genomic heterogeneity affecting diagnosis + ; Presence of intratumoral genomic heterogeneity affecting diagnosis (the number of clones different from others)

* Intestinal or diffuse type the mini-array was evaluated for a blinded set of 30 gastric cancers independent of the training set of 83 cases.

The mini-array allows for the automatic differentiation between the tumors with and those without a specific characteristic. The overall accuracy for the blinded-sample set was $66.7 \%$ (sensitivity; 0.95 , and specificity; 0.0 ) for node metastasis, $86.7 \%$ (sensitivity; 0.67 and specificity; 0.85 ) for liver metastasis, $86.7 \%$ (sensitivity; 0.67 , and specificity; 0.89 ) for peritoneal dissemination, and $96.7 \%$ (sensitivity; 0.20, and specificity 1.0) for the depth of tumor invasion. The mini-array provides a guideline for the optimal treatment of each patient with gastric cancer. Early cancers with a node-negative status are an indication for EMR or ESD. Therefore, information concerning the nodal state and the depth of tumor invasion is primarily essential for the determination of the treatment methods and can improve the quality of life for cancer patients. In the differentiation between the early and advanced cancers, the diagnostic accuracy was as high as $96.7 \%$ (sensitivity; 1.0 , and specificity 0.67 ). However, the diagnostic accuracy for nodal metastasis was not high; the false positive and false negative rates were $100 \%$ and 5\%, respectively. These findings indicate the validity of the practical use of the mini-array to determine the optimal treatment methods. However, the diagnostic accuracy, specificity and sensitivity of the mini-array for the blinded samples may not completely meet the requirement of surgeons at present. The mini-array needs to be revised to improve the diagnostic performance. The number of tumors examined in this study was limited, and large-scale studies may identify the BAC clones with a stronger impact on the differentiation between the tumors with and without specific features. 
CGH is technically applicable to biopsy specimens as well as surgical ones, because a sufficient amount of genomic DNA can be readily obtained from the biopsy specimens [26]. Before the application of the mini-array to the biopsy specimens, however, the issue of intratumoral heterogeneity must be investigated from the viewpoint of diagnostic accuracy [27]. The effects of the intratumoral heterogeneity on the diagnostic performance of the miniarray were examined using four tissue specimens taken from different parts of a cancer. The diagnosis by the miniarray analysis was hardly different between the four tissue specimens taken from a tumor. This indicates that biopsy specimens as well as surgical ones can be applied to the mini-array for the purpose of estimating the disease states of gastric cancer. Therefore, the mini-array can be added to a list of the laboratory examinations which can be used for the diagnosis of gastric tumors and to also select the optimal therapy.

\section{Conclusion}

We made the mini-array in order to estimate the degree of node metastasis, liver metastasis, peritoneal dissemination, depth of mural invasion, and the histologic type of gastric adenocarcinoma with a high degree of accuracy.

\section{Abbreviations}

BAC: bacterial artificial chromosome; CGH: comparative genomic hybridization; EMR: endoscopic mucosal resection; ESD: endoscopic submucosal dissection.

\section{Competing interests}

The authors declare that they have no competing interests.

\section{Authors' contributions}

TM, SK and TA were responsible for the majority of the molecular genetic studies involving the screening of the gastric adenocarcinomas. TU and AA collected the patient samples and the clinical data in the study. MN and AO did the statistical analyses. KS participated in the study design and helped in drafting the manuscript. SJY contributed the preparation of the mini-array.

The authors read and approved the final manuscript.

\section{Additional material}

\section{Additional file 1}

Array CGH data. This file includes the array CGH data for 83 gastric cancers.

Click here for file

[http://www.biomedcentral.com/content/supplementary/14712407-8-393-S1.xls]

\section{Acknowledgements}

Grant support: The New Energy and Industrial Technology Development Organization (NEDO) of Japan and The Ministry of Education, Culture of Japan (I5659087, 16390107, and 19390I02)

\section{References}

I. Brennan MF: Current status of surgery for gastric cancer: a review. Gastric Cancer 2005, 8:64-70.

2. Gotoda T: Endoscopic resection of early gastric cancer. Gastric Cancer 2007, 10:I-II.

3. Solinas-Toldo S, Lampel S, Stilgenbauer S, Nickolenko J, Benner A, Dohner H, Cremer T, Lichter P: Matrix-based comparative genomic hybridization: Biochips to screen for genomic imbalances. Genes Chromosomes Cancer 1997, 20:399-407.

4. Tay ST, Leong SH, Yu K, Aggarwal A, Tan SY, Lee CH, Wong K, Visvanathan J, Lim D, Wong WK, Soo KC, Kon OL, Tan P: A combined comparative genomic hybridization and expression microarray analysis of gastric cancer reveals novel molecular subtypes. Cancer Res 2003, 63:3309-16.

5. Peng DF, Sugihara H, Mukaisho K, Ling ZQ, Hattori T: Genetic lineage of poorly differentiated gastric carcinoma with a tubular component analysed by comparative genomic hybridization. J Pathol 2004, 203:884-95.

6. Weiss MM, Kuipers EJ, Postma C, Snijders AM, Pinkel D, Meuwissen SG, Albertson D, Meijer GA: Genomic alterations in primary gastric adenocarcinomas correlate with clinicopathological characteristics and survival. Cell Oncol 2004, 26:307-I7.

7. Weiss MM, Snijders AM, Kuipers EJ, Ylstra B, Pinkel D, Meuwissen SG, van Diest PJ, Albertson DG, Meijer GA: Determination of amplicon boundaries at $20 \mathrm{q} / 3.2$ in tissue samples of human gastric adenocarcinomas by high-resolution microarray comparative genomic hybridization. J Pathol 2003, 200:320-6.

8. Tsubosa Y, Sugihara H, Mukaisho K, Kamitani S, Peng DF, Ling ZQ, Tani T, Hattori T: Effects of degenerate oligonucleotideprimed polymerase chain reaction amplification and labeling methods on the sensitivity and specificity of metaphase- and array-based comparative genomic hybridization. Cancer Genet Cytogenet 2005, I 58: 156-66.

9. Gorringe KL, Boussioutas A, Bowtell DD, Melbourne Gastric Cancer Group, Peter Mac Micro Array Facility: Novel regions of chromosomal amplification at $6 \mathrm{p} 21,5 \mathrm{p} 13$, and $12 \mathrm{q} 14$ in gastric cancer identified by array comparative genomic hybridization. Genes Chromosomes Cancer 2005, 42:247-59.

10. Hashimoto Y, Oga A, Okami K, Imate Y, Yamashita Y, Sasaki K: Relationship between cytogenetic aberrations by $\mathrm{CGH}$ coupled with tissue microdissection and DNA ploidy by laser scanning cytometry in head and neck squamous cell carcinoma. Cytometry 2000, 40:161-166.

II. Kong G, Oga A, Park CK, Kawauchi S, Furuya T, Sasaki K: DNA sequence copy number aberrations associated with histological subtypes and DNA ploidy in gastric carcinoma. Jpn J Cancer Res 200I, 92:740-747.

12. Oga A, Kong G, Ishii Y, Izumi H, Park CY, Sasaki K: Preferential loss of 5q 14-2I in intestinal-type gastric cancer with DNA aneuploidy. Cytometry 2001, 46:57-62.

13. Furuya T, Uchiyama T, Adachi A, Chochi Y, Oga A, Kawauchi S, Ishiglo $\mathrm{K}$, Kohsuke Sasaki K: Relation of DNA ploidy to genetic aberrations detected by chromosomal CGH and FISH in gastric adenocarcinomas. Oncol Rep 2006, I5:|49|-6.

14. Cho YL, Bae S, Koo MS, Kim KM, Chun HJ, Kim CK, Ro DY, Kim JH, Lee CH, Kim YW, Ahn WS: Array comparative genomic hybridization analysis of uterine leiomyosarcoma. Gynecol Oncol 2005, 99:545-5I.

15. Ohguri T, Hisaoka M, Kawauchi S, Sasaki K, Aoki T, Kanemitsu S, Matsuyama $A$, Korogi $Y$, Hashimoto $H$ : Cytogenetic analysis of myxoid liposarcoma and myxofibrosarcoma by array-based comparative genomic hybridization. I Clin Pathol 2006, 59:978-83.

16. Yamamoto $\mathrm{Y}$, Chochi $\mathrm{Y}$, Matsuyama H, Eguchi S, Kawauchi S, Furuya T, Oga A, Kang JJ, Naito K, Sasaki K: Gain of 5p I 5.33 Is Associated with Progression of Bladder Cancer. Oncology 2007, 72:132-138. 
17. Witten IH, Frank E: Data Mining: Practical Machine Learning Tools and Techniques with Java Implementations. Morgan Kaufman: San Francisco; 2000.

18. Hidaka S, Yasutake T, Kondo M, Takeshita H, Yano H, Haseba M, Tsuji T, Sawai T, Nakagoe T, Tagawa Y: Frequent gains of 20q and losses of $18 \mathrm{q}$ are associated with lymph node metastasis in intestinal-type gastric cancer. Anticancer Res 2003, 23:3353-7.

19. Kimura Y, Noguchi T, Kawahara K, Kashima K, Daa T, Yokoyama S: Genetic alterations in 102 primary gastric cancers by comparative genomic hybridization: gain of $20 q$ and loss of $18 q$ are associated with tumor progression. Mod Pathol 2004, 17:1328-37.

20. Morohara K, Nakao K, Tajima Y, Nishino N, Yamazaki K, Kaetsu T, Suzuki S, Tsunoda A, Kawamura M, Aida T, Tachikawa T, Kusano M: Analysis by comparative genomic hybridization of gastric cancer with peritoneal dissemination and/or positive peritoneal cytology. Cancer Genet Cytogenet 2005, 16 1:57-62.

21. Noguchi T, Wirtz HC, Michaelis S, Gabbert HE, Mueller W: Chromosomal imbalances in gastric cancer. Correlation with histologic subtypes and tumor progression. Am J Clin Pathol 200I, I I 5:828-34.

22. Gutman S, Kessler LG: The US Food and Drug Administration perspective on cancer biomarker development. Nat Rev Cancer 2006, 6:565-7I.

23. Vijver MJ van de, He YD, van't Veer LJ, Dai H, Hart AA, Voskuil DW, Schreiber GJ, Peterse JL, Roberts C, Marton MJ, Parrish M, Atsma D, Witteveen A, Glas A, Delahaye L, Velde T van der, Bartelink $H$, Rodenhuis S, Rutgers ET, Friend SH, Bernards R: A gene-expression signature as a predictor of survival in breast cancer. $\mathrm{N}$ Engl J Med 2002, 347: 1999-2009.

24. van 't Veer LJ, Dai $H$, Vijver MJ van de, He YD, Hart AA, Mao M, Peterse HL, Kooy K van der, Marton MJ, Witteveen AT, Schreiber G], Kerkhoven RM, Roberts C, Linsley PS, Bernards R, Friend SH: Gene expression profiling predicts clinical outcome of breast cancer. Nature 2002, 4I5:530-6.

25. Glas AM, Floore A, Delahaye LJ, Witteveen AT, Pover RC, Bakx N, Lahti-Domenici JS, Bruinsma T], Warmoes MO, Bernards R, Wessels LF, Van't Veer LJ: Converting a breast cancer microarray signature into a high-throughput diagnostic test. BMC Genomics 2006, 7:278.

26. Ueno T, Tangoku A, Yoshino S, Abe T, Hayashi H, Toshimitsu H, Hashimoto K, Satoh T, Oga A, Furuya T, Oka M, Sasaki K: Prediction of Nodal Metastasis by Comparative Genomic Hybridization in Biopsy Specimens from Patients with Superficial Esophageal Squamous Cell Carcinoma. Clin Cancer Res 2003, 9:5|37-5|4|.

27. Harada T, Okita K, Shiraishi K, Kusano N, Kondoh S, Sasaki K: Interglandular cytogenetic heterogeneity detected by comparative genomic hybridization in pancreatic cancer. Cancer Res 2002, 62:835-9.

\section{Pre-publication history}

The pre-publication history for this paper can be accessed here:

http://www.biomedcentral.com/1471-2407/8/393/pre pub
Publish with Biomed Central and every scientist can read your work free of charge

"BioMed Central will be the most significant development for disseminating the results of biomedical research in our lifetime. "

Sir Paul Nurse, Cancer Research UK

Your research papers will be:

- available free of charge to the entire biomedical community

- peer reviewed and published immediately upon acceptance

- cited in PubMed and archived on PubMed Central

- yours - you keep the copyright

Submit your manuscript here:

http://www.biomedcentral.com/info/publishing_adv.asp
BiolMedcentral 